\title{
NONARTHRITIC HIP SCORE: TRANSLATION, CULTURAL ADAPTATION AND VALIDATION OF THE ITALIAN VERSION
}

\author{
Martina PANICO ${ }^{1}$, Giovanni GALEOTO ${ }^{2}$, Vittorio BARTOLI ${ }^{3}$, Josephine VALENTINO ${ }^{3}$, \\ Anna BERARDI ${ }^{1}$, Marco TOFANI ${ }^{4}$, Fabrizio MAGNIFICA ${ }^{3,5}$
}

1. Sapienza University of Rome, Rome, Italy

2. Department of Public Health and Infectious Diseases, Sapienza University of Rome, Rome Italy

3. Italian Air Force Aerospace Medicine Department, Diagnostic Therapeutic and Rehabilitative Aeromedical Center, Rome, Italy

4. Neurorehabilitation Unit, Department of Neurosciences and Neurorehabilitation, Bambino Gesù Children's Hospital, Italy

5. Department of Human Neurosciences, Sapienza University of Rome, Rome, Italy

*Corresponding author: Fabrizio Magnifica, Italian Air Force Aerospace Medicine Department, Diagnostic Therapeutic and Rehabilitative Aeromedical Center, Rome, Italy +390649866652 - +393337170700 Viale Piero Gobetti 6A, fabrizio.magnifica@uniroma1.it

\begin{abstract}
BACKGROUND: the aim of the study was to translate the Nonarthritic Hip Score (NAHS) questionnaire into the Italian language, to adapt it into the Italian culture and to validate it.

METHODS: Translation and cultural adaptation were performed following international guidelines. To assess the reliability of the tool internal consistency and test-retest reliability were evaluated through Cronbach's alpha and Intraclass Correlation Coefficient (ICC) respectively. Validity was evaluated by Pearson's correlation coefficient between the NAHS, the Lequesne Index of Severity for Osteoarthritis of the Hip (LISOH) and the Western Ontario and McMaster Universities Arthritis Index (WOMAC), that were administered together. These three questionnaires were applied to a population of 64 individuals between 19 and 82 years old with generic hip injures or diseases.
\end{abstract}


RESULTS: The items of the original version of NAHS are similar or equal to the items of the Italian version of NAHS. Cronbach's $\alpha$ was 0.614 (Pain); 0.821 (Symptom); 0.877 (Function); 0.896 (Activity). ICC for total score was 0.905. The correlation with the WOMAC was $0.850(\mathrm{p}<0.01)$ and with the LISOH was $0.738(\mathrm{p}<0.01)$.

CONCLUSIONS: The NAHS showed positive results for reliability and validity so it was translated into Italian language and was cross-culturally adapted to Italian culture. This questionnaire is a good instrument in clinical practice and scientific research for the evaluation of young and active individuals affected by hip injures or diseases.

Key words: hip, pain, young, osteoarthritis

\section{Introduction}

The Nonarthritic Hip Score (NAHS) was designed for the evaluation of joint diseases in young individuals with high physical demands and high treatment expectations. Before it the development scales designed for the evaluation of hip injures and disorders were created only for older people, these scales focused their attention on arthritic and degenerative joint disease. So these scores were not appropriate to assess the younger and active population of people that have not severe hip injures or diseases [1]. The NAHS is a scale for the evaluation of functional capacity of the hip in young and physically active individuals. It is a simple, short and self-administered questionnaire composed of 20 multiple choice questions divided into 4 sections: pain (5 components), function (4 components), mechanical symptoms (5 components) and physical activity level (6 components). Every question has the same five responses and each of the answers corresponds to a particular numerical value. The total NAHS score is calculated by adding together the values and multiplying by 1.25 to obtain the final score. The maximum score is 100 corresponding to a normal hip function [1]. The NAHS was developed in 2003 by Christian P. Christensen et al. in English language and it was also translated, validated and cross-cultural adapted in Portuguese language in Brazil in 2013[2]. The aim of this study was to translate, culturally adapt and validate NAHS into Italian Language. The most used scales for the evaluation of hip disorders that have been translated and validated into Italian language as the Harris Hip Score, Lequesne Index 
of Severity for Osteoarthritis of the Hip (LISOH) and the Western Ontario and McMaster Universities Arthritis Index (WOMAC) [3] are appropriate only for individuals with moderate to severe hip osteoarthritis or with post-traumatic hip disorders with significant physical limitations. Thus with the increasing of hip diseases [2] between young individuals Italian clinicians and researchers had the need to validate a questionnaire for this population that still not have several functional limitations.

\section{Materials and methods}

Translation and cultural adaptation. Once the consent of the developers of the Nonarthritic Hip Score (NAHS) was received, following the "Translation and Cultural Adaptation of Patient-Reported Outcomes Measures-Principles of Good Practice" guidelines the original tool was translated from English to Italian. The original English version of the NAHS was translated by two Italian physiotherapists familiar with English. Two English translators then translated the scales back into English. Finally a group of two Italian physiotherapists worked for the cultural adaptation, compared the original version with the back translations and they checked the final translation and corrected any remaining error to minimize any differences from the English original version.

Participants. According to preceding validations of the NAHS [1, 2] participants included in the study had to be aged 18 to 76 and they had to be affected by hip injures or diseases for 6 months. Patients were excluded from the study if they had: visual or cognitive problems, severe functional hip limitations with decreased range of motion (less than $10^{\circ}$ of internal rotation at $90^{\circ}$ hip flexion), severe osteoarthritis, in treating for any therapy. All the participants included in the study gave their consent for the study.

Reliability and Validity. Following the "Consensus-Based Standards for the Selection of Health Status Measurement Instruments" (COSMIN) checklist, the reliability and validity of the culturally adapted scale were assessed. The internal consistency of NAHS was examined by Cronbach's alpha $(\alpha)$, needed to be $>0.70$. To calculate test-retest reliability the patients completed the NAHS after 48 hours. The questionnaires were given by one physiotherapist. NAHS-retest was administered after 48 hours. Test- retest reliability was measured with the Intraclass Correlation Coefficient (ICC). With an ICC> 0.70 NAHS was considered stable.The participants completed the Italian version of the NAHS, the LISOH [4-7] and the WOMAC [8-9] questionnaires. 
Validity was assessed using Pearson's correlation analyses to determine the association between NAHS and the Italian version of WOMAC and LISOH. Person's correlation coefficient needed to be $>0.5$ or $<-0.5$, where positive values show positive linear correlation and negative values show negative linear correlation. The significance level has been set for p-value less than or equal to 0.05 . All statistical analyses were performed using IBM-SPSS version 23.00 .

\section{Results}

Translation and cultural adaptation. The final Italian translation of NAHS was done following the guidelines for translation and cultural adaptation, elaborating a forward and a backward translation. Finally the Italian version of the (NAHS-I) was formed by all the items identical or similar to the original version.

Participants. Participants were recruited from April 2019 through "Policlinico Umberto I" and "Policlinico Italia", "Aeronautica Militare". Among the 64 patients recruited all agreed to participate (mean age \pm standard deviation $(\mathrm{SD})=48.48 \pm 16,05)$ and completed the study without any loss. The characteristics of the participants are summarized in Table I. Reliability. The NAHS-I showed a good level of internal consistency with a Cronbach's $\alpha$ of 0.614 (“Pain”); 0.821 (“Symptom”); 0.877 (“Function”); 0.896 (“Activity”) (Table II).

NAHS was administered for the second time after 48 hours from the first administration and as reported in table in Table III it was reliable with an ICC of $0.905(\mathrm{p}<0.05)$ and the scale remains stable also after the second administration to the same individual.

Validity. The Italian versions of WOMAC and LISOH were also administered to the population. The Pearson's correlation coefficient of the total score of the NAHS with the Italian version of the WOMAC was $0.850(\mathrm{p}<0.01)$ and with the Italian version of the LISOH was $0.738(\mathrm{p}<0.01)$, proving that NAHS has a good validity and that it has all statistically significant correlations with the other two scales (Table IV).

\section{Discussion}

The Nonarthritic Hip Score (NAHS) has been translated and culturally adapted into Italian language and culture. The NAHS is a very simple self-administered questionnaire and it request really short time to fill in. For this reasons it is a very good and practical instrument for the evaluation of patients in clinical practice and in research studies. 
The instruments for the evaluation of patients affected by hip diseases available in the last century were developed for patients with osteoarthritis of the hip or subsequent to hip arthroplasty. So these scales were not appropriate enough for young and active patients with high physical demands. The most used scales for the evaluation of hip diseases, like the "Harris Hip Score"[10] and the "WOMAC" [8-9] focused their attention on older people predominantly affected by osteoarthritis. For this reason we have chosen a lower mean age of the patients (48.48) and patients affected by severe osteoarthritis were excluded compared to the validations of the LINOH [4-7] and WOMAC [8-9] scales. The LINOH's evaluation skills were evaluated [5-6], but the sections of the scale have not been validated for independent administration [7]. Also in the Italian validation of the WOMAC [8] the Lequesne OA index was used as a comparison scale and it was proposed as an interview technique [7].

NAHS is composed only by twenty multiple choice questions, each one with the same five potential answers. The ten questions relating with pain and function come directly from WOMAC [8-9]. The other ten questions (six relating activity level and four relating mechanical symptom) were chosen after a long consultation with patients. The authors supported that only six questions about activity level and only four for mechanical symptom were enough to assess this kind of patients and to catch the disease [1].

Comparison with other studies. The results of this study demonstrate, evaluating Validity and Reliability, that the Italian version of the NAHS is a reliable and valid instrument of measure with good evaluation skills. Values of Cronbach $\alpha$ are good for all NAHS's sections, except for the first section. This demonstrate that the NAHS has a great internal consistency and a good interrelationship between items. One of the possible reasons of the not statistically significance of the first section of the NAHS could be the fact that the sample was composed only by 64 individuals. Furthermore we can see in all the items are important, because if an item were eliminated the value of the Cronbach's $\alpha$ would decrease and also the internal consistency would diminish. The Intraclass Correlation Coefficient (ICC) is $>0.7$, so NAHS has a great stability and also with the administration of a second questionnaire after 48 hours, the instrument continues to be stable. As reported in Table 3, the NAHS was compared with the WOMAC [8] and LINOH [5-6] scales. The lower value of the Pearson's correlation coefficient was 0.341 between the fourth NAHS's section(“Activity") and the second WOMAC's section ("Stiffness"). The high value of the Pearson's correlation coefficient between NAHS and WOMAC scores (0.850) demonstrate 
that both scales asses similar features and show a close relation between them. This can be explained by the fact that ten NAHS's questions came directly from WOMAC. The evaluation of the Pearson's correlation coefficient demonstrates all statistically significant correlations with both scales, so all NAHS's items are correlated with all WOMAC's and LINOH items.

Limitations of the study. One limitation of the scale is the need of the research's participation in questionnaire administration to the individuals with low educational levels. Another limitation of the NAHS could be the short time (48 hours) between the two tests, because the patient could remember the answers of the first test administration. The limitation of the study is the small sample of individuals (64).

\section{Conclusions}

This study showed that the NAHS is considered a good instrument for the evaluation of young and active patients with hip disease. NAHS is a good instrument in clinical practice and in research studies because it is very simple, self- administered and quick to complete (only 5 minutes) and to understand for a maximum compliance of the patient and it is specific for young and physically active individuals [11-22].

This study was conducted by a group of rehabilitation professionals from the "Sapienza" University of Rome, from "Rehabilitation and Outcome Measure Assessment" (ROMA) association. Even rehabilitation professionals from the Aerospace Medicine Department of the Italian Air Force of the Diagnostic Therapeutic Center and aero-medical rehabilitation were part of the team research who conducted the study. ROMA association in the last few years has dealt with the validation of many outcome measures in Italy.

\section{REFERENCES}

1. Christensen C.P., Althausen P.L., Mittleman M.A., Lee J.A., McCarthy J.C. The nonarthritic hip score: reliable and validated. Clin Orthop Relat Res, 2003; (406):75-83. DOI: 10.1097/01.blo.0000043047.84315.4b.

2. Del Castillo LN, Leporace G, Cardinot TM, Levy RA, Oliveira LP. Translation, crosscultural adaptation and validation of the Brazilian version of the Nonarthritic Hip Score. Sao Paulo Med J. 2013; 131(4):244-51. doi: 10.1590/1516-3180.2013.1314487. 
3. A. Nilsdotter, A.Bremander. Measures of hip function and symptoms: Harris Hip Score (HHS), Hip Disability and Osteoarthritis Outcome Score (HOOS), Oxford Hip Score (OHS), Lequesne Index of Severity for Osteoarthritis of the Hip (LISOH), and American Academy of Orthopedic Surgeons (AAOS) Hip and Knee Questionnaire. Arthritis Care Res (Hoboken), 2011 Nov; 63 (11):S200-7. doi: 10.1002/acr.20549.

4. Dawson J, Linsell L, Doll H, Zondervan K, Rose P, Carr A, Randall T, Fitzpatrick R. Assessment of the Lequesne index of severity for osteoarthritis of the hip in an elderly population. Osteoarthritis Cartilage, 2005 Oct; 13(10):854-60. DOI: 10.1016/j.joca.2005.05.006.

5. Lequesne MG, Mery C, Samson M, Gerard P. Indexes of severity for osteoarthritis of the hip and knee. Validation--value in comparison with other assessment tests. Scand J Rheumatol Suppl, 1987; (65):85-9. PMID: 3479839

6. Lequesne MG, Samson M. Indices of severity in osteoarthritis for weight bearing joints. J Rheumatol Suppl, 1991 Feb; (27):16-8. PMID: 2027118.

7. G. Stucki, O. Sangha, S. Stucki, B.A. Michel, A. Tyndall, W. Dick, et al. Comparison of the WOMAC (Western Ontario and McMaster Universities) osteoarthritis index and a selfreport format of the self-administered Lequesne-Algofunctional index in patients with knee and hip osteoarthritis. Osteoarthritis Cartilage, 1998 Mar; 6(2):79-86. DOI: 10.1053/joca.1997.0097.

8. Salaffi F, Leardini G, Canesi B, Mannoni A, Fioravanti A, Caporali R, Lapadula G, Punzi L; GOnorthrosis and Quality Of Life Assessment (GOQOLA). Reliability and validity of the Western Ontario and McMaster Universities (WOMAC) Osteoarthritis Index in Italian patients with osteoarthritis of the knee. Osteoarthritis Cartilage, 2003 Aug; 11(8):551-60. PMID: 12880577.

9. Bellamy N, Watson Buchanan W, Goldsmith CH, Campbell J, Stitt LW. Validation study of WOMAC: a health status instrument for measuring clinically important patient relevant outcomes to antirheumatic drug therapy in patients with osteoarthritis of the hip or the knee. J Rheumatol, 1988; (15):1833-40. PMID: 3068365.

10. Dettoni F1, Pellegrino P, La Russa MR, Bonasia DE, Blonna D, Bruzzone M, Castoldi F, Rossi R. Validation and cross cultural adaptation of the Italian version of the Harris Hip Score. Hip Int, 2015 Jan-Feb; 25(1):91-7. doi:10.5301/hipint.5000184. 
11. Berardi A., Biondillo A., Màrquez M. A., De Santis R., Fabbrini G., Tofani M. et al. Validation of the short version of the Van Lieshout Test in an Italian population with cervical spinal cord injuries: a cross-sectional study. Spinal cord. 2019; 57(4): 339.

12. Galeoto G., Scialpi A., Grassi M. L., Berardi A., Valente D., Tofani M., et al. General Sleep Disturbance Scale: Translation, cultural adaptation, and psychometric properties of the Italian version. CRANIO®. 2019; 1-9.

13. Savona A., Ferralis L., Saffioti M., Tofani M., Nobilia M., Culicchia G. et al. Evaluation of intra-and inter-rater reliability and concurrent validity of the Italian version of the Jebsen-Taylor Hand Function Test in adults with rheumatoid arthritis. Hand Therapy. 2019; 24(2): 48-54.

14. Berardi A., Dhrami L., Tofani M., Valente D., Sansoni J., Galeoto G. Cross-cultural adaptation and validation in the Italian population of the wolf motor function test in patients with stroke. Functional neurology. 2018. 33(4): 229-253.

15. Dattoli S., Colucci M., Soave M. G., De Santis R., Segaletti L., Corsi C. et al. Evaluation of pelvis postural systems in spinal cord injury patients: Outcome research. $\mathrm{J}$ Spinal Cord Med. 2018; 1-13.

16. Galeoto G., Colucci M., Guarino D., Esposito G., Cosma E., De Santis R. et al. Exploring validity, reliability, and factor analysis of the Quebec User Evaluation of Satisfaction with Assistive Technology in an Italian Population: a cross-sectional study. Occup Ther Health Care. 2018; 32(4): 380-392.

17. Galeoto G., Berardi A., Pizzi C., Farina I., Sansoni J. Evaluation of the Disciplinary Competences of the Students of the Bachelor's Degree in Physiotherapy at "Sapienza" University of Rome Through the TECO: A Cross-Sectional Study. In International Workshop on Learning Technology for Education in Cloud (pp. 93-102). Springer, Cham; 2019

18. Covotta A., Gagliardi M., Berardi A., Maggi G., Pierelli F., Mollica R. et al. Physical Activity Scale for the elderly: translation, cultural adaptation, and validation of the Italian version. Curr Gerontol Geriatr Res. 2018: 8294568.

19. Galeoto G., Iori F., De Santis R., Santilli V., Mollica R., Marquez M. A. et al. The outcome measures for loss of functionality in the activities of daily living of adults after stroke: a systematic review. Top Stroke Rehabil.. 2019; 26(3): 236-245. 
20. Ruggieri M., Palmisano B., Fratocchi G., Santilli V., Mollica R., Berardi A. et al. Validated Fall Risk Assessment Tools for Use with Older Adults: A Systematic Review. Physical \& Occupational Therapy In Geriatrics, 36(4): 331-353.

21. Berardi A., Galeoto G., Tofani M., Mangone M., Ratti S., Danti A., Sansoni J., Marquez M. A. The Validity of Rasterstereography as a Technological Tool for the Objectification of Postural Assessment in the Clinical and Educational Fields: Pilot Study. n book: Methodologies and Intelligent Systems for Technology Enhanced Learning, 9th International Conference, Workshops, pp.55-62; 2019

22. Galeoto G., Piepoli V., Ciccone E., Mollica R., Federici C., Magnifica F., Servadio. Musculoskeletal Health Questionnaire: translation, cultural adaptation and validation of the Italian version (MSK-HQ-I). MLTJ. 295-303.

\section{NOTES}

Conflicts of interest. The authors certify that there is no conflict of interest with any financial organization regarding the material discussed in the manuscript.

Congresses. $9^{\wedge}$ I.S.Mu.L.T. Congress Open mind and new technologies in muscles, ligaments and tendons on November 29-30, 2019. 


\section{TABLES}

Table I.- Demographic characteristic of the 64 participants.

\begin{tabular}{|l|l|}
\hline & Sample ( $n=64)$ \\
\hline Age mean (standard deviation) & $48.48(16,05)$ \\
\hline Females number (\%) & $35(54.7)$ \\
\hline Diagnosis number (\%) & $1(1.6)$ \\
\hline Rheumatoid arthritis & \\
\hline Aspecific hip pain & $43(67.2)$ \\
\hline Femoroacetabular impingement & $1(1.6)$ \\
\hline Periarticular tendonitis & $2(3.1)$ \\
\hline Pertrochanteric bone cysts & $1(1.6)$ \\
\hline Onitial osteoarthritis & $12(18.75)$ \\
\hline
\end{tabular}


Table II. Internal consistency: Cronbach's alpha for the subscales and Cronbach's alpha if item deleted.

\begin{tabular}{|c|c|}
\hline & Cronbach's alpha if item deleted \\
\hline item1 & 0.458 \\
\hline item2 & 0.525 \\
\hline item3 & 0.624 \\
\hline item4 & 0.630 \\
\hline item5 & 0.541 \\
\hline total alpha "PAIN" & 0.614 \\
\hline item6 & 0.750 \\
\hline item7 & 0.778 \\
\hline item8 & 0.746 \\
\hline item9 & 0.817 \\
\hline total alpha "SYMPTOM" & 0.821 \\
\hline item10 & 0.850 \\
\hline item11 & 0.849 \\
\hline item 12 & 0.841 \\
\hline item13 & 0.865 \\
\hline item14 & 0.849 \\
\hline total alpha "FUNCTION" & 0.877 \\
\hline item15 & 0.865 \\
\hline item16 & 0.855 \\
\hline item17 & 0.875 \\
\hline item18 & 0.858 \\
\hline item19 & 0.863 \\
\hline item20 & 0.874 \\
\hline total alpha "ACTIVITY" & 0.896 \\
\hline
\end{tabular}


Table III. Stability: Intraclass correlation coefficient (ICC) between test and retest of 64 participants.

\begin{tabular}{|l|c|c|l|l|}
\hline & test & retest & & \\
\hline & Mean Score \pm Standard Deviation & Mean Score \pm Standard Deviation & $\begin{array}{l}\text { Intraclass } \\
\text { Correlation } \\
\text { Coefficient }\end{array}$ & $\begin{array}{l}\text { Confidence } \\
\text { interval } \\
\text { 95\% [lower } \\
\text { limit-upper } \\
\text { limit }\end{array}$ \\
\hline PAIN & $15.22 \pm 2.68$ & $15.22 \pm 3.12$ & 0.779 & $0.64-0.87$ \\
\hline SYMPTOM II & $11.47 \pm 3.35$ & $12.03 \pm 3.21$ & 0.858 & $0.77-0.91$ \\
\hline FUNCTION III & $15.62 \pm 3.89$ & $15.75 \pm 3.84$ & 0.933 & $0.89-0.96$ \\
\hline ACTIVITY IV & $16.23 \pm 4.63$ & $16.69 \pm 4.18$ & 0.912 & $0.85-0.95$ \\
\hline TOTAL & $73.18 \pm 15.36$ & & & $0.84-0.94$ \\
\hline
\end{tabular}


Table IV. Validity: Pearson's correlation coefficient between Western Ontario and McMaster Universities Arthritis Index (WOMAC), Lequesne Index of Severity for Osteoarthritis of the Hip (LISOH) and Nonarthritic Hip Score.

\begin{tabular}{|l|l|l|l|l|l|l|l|l|}
\hline & PAIN & STIFFNESS & PHYSICAL & TOTAL & PAIN & MAXIMUM & $\begin{array}{l}\text { ACTIVITIES OF } \\
\text { DAILY LIVING }\end{array}$ & TOTAL \\
& & & FUNCTION & & & DISTANCE & \\
Pain & $-0.694^{* *}$ & $-0.375^{* *}$ & $-0.677^{* *}$ & $-0.690^{* *}$ & $.848^{* *}$ & $0.847^{* *}$ & $-0.691^{* *}$ & $-0.542^{* *}$ \\
\hline Sympom & $-0.685^{* *}$ & $-0.551^{* *}$ & $-0.554^{* *}$ & $-0.614^{* *}$ & $.768^{* *}$ & $0.770^{* *}$ & $-0.614^{* *}$ & $-0.374^{* *}$ \\
\hline Function & $-0.629^{* *}$ & $-0.580^{* *}$ & $-0.904^{* *}$ & $-0.875^{* *}$ & $.866^{* *}$ & $0.870^{* *}$ & $-0.875^{* *}$ & $-0.794^{* *}$ \\
\hline Activity & $-0.480^{* *}$ & $-0.341^{* *}$ & $-0.718^{* *}$ & $-0.678^{* *}$ & $.879^{* *}$ & $0.875^{* *}$ & $-0.678^{* *}$ & $-0.707^{* *}$ \\
\hline TOTAL & $-0.718^{* *}$ & $-0.544^{* *}$ & $-0.855^{* *}$ & $-0.850^{* *}$ & & $10.000^{* *}$ & $-0.850^{* *}$ & $-0.738^{* *}$ \\
\hline
\end{tabular}

